\title{
Cultura e clima organizacionais: um estudo em indústrias de laticínios do Estado do Rio Grande do Norte
}

\author{
Organizational culture and climate: a study in Rio Grande do Norte's \\ dairy industries
}

\author{
Luiz Célio Souza Rocha' \\ Emanuelly Alves Pelogio² \\ Miguel Eduardo Moreno Añez ${ }^{3}$
}

\begin{abstract}
Resumo: O objetivo deste trabalho é investigar se existe relação entre a cultura organizacional e o clima organizacional, tendo, como locus de pesquisa, indústrias de laticínios do Rio Grande do Norte. Para tanto, realizou-se um estudo exploratório-descritivo e conclusivo-causal, em uma amostra composta por 211 funcionários de todos os níveis hierárquicos das empresas participantes, o que corresponde a $25,6 \%$ do total do setor. A partir dos dados coletados, identificaram-se as características pessoais dos respondentes, o perfil da cultura organizacional predominante e o clima organizacional predominante nas indústrias analisadas. Para a análise da cultura organizacional, utilizou-se o Modelo dos Valores Competitivos (CAMERON; QUINN, 2006) com adaptações, segundo Santos (2000). Para a análise do clima organizacional, utilizou-se a Escala de Medida de Clima Organizacional, proposta por Martins et al. (2004) e Martins (2008), com modificações de forma a se ter um instrumento mais enxuto. Os dados coletados foram submetidos a análises estatísticas quantitativas que permitiram chegar às seguintes conclusões: os perfis culturais foram encontrados de forma equilibrada nas organizações pesquisadas, com ênfase para os perfis “cultura clã" e "cultura mercado"; as organizações pesquisadas possuem um clima bom, segundo a classificação de Martins (2008), com destaque para os fatores "apoio da chefia e da organização" e "conforto físico", fatores estes bem coerentes com o perfil "cultura clã"; e os perfis culturais apresentaram influência sobre os fatores do clima organizacional. Assim, os resultados nos permitem concluir que há relações entre os perfis culturais e os fatores determinantes do clima organizacional nas organizações pesquisadas.
\end{abstract}

Palavras-chave: Cultura organizacional. Clima organizacional. Indústrias de laticínios.

\begin{abstract}
The objective of this study is to investigate the relationship between organizational culture and the organizational climate in Rio Grande do Norte's dairy industries. An exploratory-descriptive and conclusive-causal study was carried out to examine a sample composed of 211 employees of all hierarchical levels of firms investigated, corresponding to $25.6 \%$ of the total of this sector. Based on the data collected, the employees' personal characteristics, the predominant organizational culture profile, and the predominant organizational climate in the industries researched were identified. In order to analyze the organizational culture, the Competing Value Model (CAMERON; QUINN, 2006), with adaptations by Santos (2000) was used. In order to analyze the organizational climate, the Organizational Climate Measurement Scale, proposed by Martins et al. (2004) and Martins (2008), with modifications, was used. The data were submitted to quantitative statistical analyses that led to the following conclusions: the organizations' cultural profiles were considered well-balanced, with emphasis to "clan culture" and "market culture" profiles; the organizational climate was considered good, based on the Martins'(2008) classification, with emphasis to "support from the top management and organization" and "physical comfort", factors that are in agreement with the "clan culture" profile; and the cultural profiles showed influence on the organizational climate factors. Therefore, based on the results, it can be concluded that there are relationships between the cultural profiles and the organizational climate factors in the organizations studied.
\end{abstract}

Keywords: Organizational culture. Organizational climate. Dairy industries.

\footnotetext{
${ }^{1}$ Instituto Federal de Educação, Ciência e Tecnologia do Sul de Minas Gerais - IFSULDEMINAS, Rod. Machado-Paraguaçú, Km 3, Santo Antônio, CEP 37750-000, Machado, MG, Brasil, e-mail: luiz.rocha@mch.ifsuldeminas.edu.br

2 Instituto Machadense de Ensino Superior - IMES, Rod. BR 267, Km 03, Parque Industrial, CEP 37750-000, Machado, MG, Brasil, e-mail: manu-pelagio@bol.com.br

${ }^{3}$ Programa de Pós-graduação em Administração - PPGA, Universidade Federal do Rio Grande do Norte - UFRN, Av. Senador Salgado Filho, s/n, Edifício do CCSA, Campus da UFRN, Lagoa Nova, CP 1570, CEP 59072-970, Natal, RN, Brasil, e-mail: anez1957@yahoo.com.br
}

Recebido em 11/4/2011 — Aceito em 3/7/2012

Suporte financeiro: Nenhum. 


\section{Introdução}

As atitudes dos funcionários e a capacidade destes de desenvolverem suas atividades em um nível de qualidade compatível com as exigências do mercado estão diretamente relacionadas à competitividade de uma organização. Segundo Costa (1999), administrar o contingente humano de uma organização e conduzi-lo ao sucesso exige que os processos de inovação e mudança sejam implementados sob estratégias de gerenciamento, as quais tenham uma atuação consistente sobre os valores desenvolvidos dentro da cultura da organização, uma vez que estes próprios valores foram desenvolvidos pelo grupo ao aprender como lidar com seus problemas de adaptação externa e integração interna, funcionando suficientemente bem para serem considerados válidos e repassados aos novos membros do grupo (SCHEIN, 1992).

Neste sentido, deve-se dar muita importância à cultura da organização. Ao se tornar mais que um simples fenômeno de moda (HOFSTEDE, 1997), a cultura fornece os dados oportunos sobre o funcionamento da organização e ajuda a entender a sua complexidade.

Outro fator também fundamental para se alcançar a performance pretendida inclui, exatamente, os múltiplos comportamentos individuais e coletivos que regem a atividade global da organização. Desta forma, deve-se dar atenção à qualidade das relações entre as pessoas e a organização. Segundo Thévenet (1985), o interesse pelo clima organizacional traduz esta preocupação: um bom clima resulta em boas relações internas com expectativas reciprocamente satisfeitas. Sabe-se que um dos maiores resultados das experiências realizadas na Western Electric foi o de mostrar a importância do clima organizacional para a produtividade.

Para Rizzatti (2002), estudar clima organizacional é um excelente mecanismo para se conhecer a organização e o ambiente de trabalho e, assim, transmitir aos dirigentes uma visão mais ampla da organização e não apenas uma simples interpretação de dados. Sob esse enfoque, o estudo do clima organizacional, seja por meio do estudo das percepções ou das aspirações dos indivíduos, é um diagnóstico da instituição, especialmente pelo seu valor prático na adoção de novas estratégias administrativas.

Mediante a possibilidade de ganhos de desempenho organizacional com a adequada gestão da cultura e do clima organizacionais (CAMERON; QUINN, 2006; DEAL; KENNEDY, 1982; HOFSTEDE et al., 1990; KOTTER; HESKETT, 1994; LIKERT, 1961 apud WALLACE, HUNT, RICHARDS, 1999; MUSIELLO NETO, 2002; PETERS; WATERMAN, 1982; SANTOS, 1998, 1999, 2000; SOUZA, 1978), faz-se necessário conhecer, empiricamente, se existe relação entre esses dois construtos e como esta ocorre nas organizações.

Assim, o presente estudo, que trata do tema "clima e cultura organizacionais", possui o seguinte problema de pesquisa: existe relação entre a cultura organizacional e o clima organizacional, ao se analisar indústrias de laticínios do Rio Grande do Norte?

Como objetivos específicos do presente trabalho, tem-se: identificar o perfil de cultura organizacional predominante nas indústrias de laticínios pesquisadas; identificar o tipo de clima organizacional predominante nas indústrias de laticínios pesquisadas; e medir, nas indústrias em foco, o grau das relações existentes entre os perfis da cultura organizacional e os fatores do clima organizacional.

\section{Cultura organizacional}

A literatura especializada está repleta de obras demonstrando particularidades marcantes entre diversas organizações existentes. Um dos aspectos mais importantes que provoca diferenças nestas instituições refere-se à cultura organizacional de cada uma delas.

Para Cameron e Quinn (2006, p. 17),

[...] cada cultura é, geralmente, refletida pela linguagem única, símbolos, regras e sentimentos etnocêntricos... Uma cultura da organização é refletida pelo que é valorizado, os estilos de liderança dominantes, a linguagem e os símbolos, os procedimentos e rotinas, e as definições de sucesso que fazem uma organização única.

Deal e Kennedy (1982) citam os valores, heróis, ritos, rituais e comunicações como elementos da cultura organizacional. Além disso, defendem que "uma cultura forte é um sistema de regras informais que indicam como as pessoas têm que se comportar na maior parte do tempo" (DEAL; KENNEDY, 1982, p. 15).

Peters e Waterman (1982) comentam que, no sentido organizacional, as "estórias, mitos ou lendas parecem ser muito importantes, pois transmitem os valores compartilhados pela organização, ou, a sua cultura" (PETERS; WATERMAN, 1982, p. 75).

Para Souza (1978, p. 36), "cultura organizacional é o conjunto de fenômenos resultantes da ação humana, visualizada dentro das fronteiras de um sistema".

Santos (2000) define cultura organizacional como sendo os valores e crenças compartilhados pelos membros de uma organização, a qual funciona como um mecanismo de controle organizacional, informalmente aprovando ou proibindo comportamentos e que dá significado, direção e mobilização para os membros da organização.

Freitas (2005, p. 97) entende a cultura organizacional: 
[...] primeiro como instrumento de poder; segundo, como conjunto de representações imaginárias sociais que se constroem e reconstroem nas relações cotidianas dentro da organização e que se expressam em termos de valores, normas, significados e interpretações, visando um sentido de direção e unidade, tornando a organização fonte de identidade e de reconhecimento para seus membros.

A autora ainda cita que um dos conceitos mais ricos na literatura sobre cultura organizacional (FREITAS, 1991) é o apresentado por Schein (1992, p. 12):

[...] um conjunto de pressupostos básicos compartilhados que um grupo aprendeu ao lidar com seus problemas de adaptação externa e de integração interna, e que funcionam suficientemente bem para serem considerados válidos e, por isso, ensinados a novos membros como a forma correta de perceber, pensar e sentir, em relação a esses problemas.

Já Hofstede (1997) define o termo, ao fazer analogia com o termo "cultura", como sendo a programação coletiva da mente que distingue os membros de uma organização dos de outra. Cita ainda que o conceito de

[...] cultura de empresa é um conceito 'suave', intangível e holístico, com, no entanto, presumíveis consequências 'duras', tangíveis [...] (HOFSTEDE, 1997, p. 34).

Menciona, também, serem esses os "ativos psicológicos" de uma organização.

Por constituir um tema bastante subjetivo, tem-se o esforço dos acadêmicos em desenvolver diversos modelos, tipologias e identificar um conjunto de dimensões que em muito ajudam a organizar os atributos fundamentais do conceito. Entretanto, não há consenso quanto à metodologia de análise que mais se adéqua ao estudo de cultura organizacional, o que gera diferentes maneiras de analisar este construto. As duas principais vertentes epistemológicas de análise da cultura organizacional (SMIRCICH, 1983; CAMERON; QUINN, 2006) são: a funcionalista, que considera a cultura organizacional como algo que a organização possui (variável); e a interpretativista, que considera a cultura organizacional como algo que a organização é (metáfora). Assim, a vertente epistemológica funcionalista de análise da cultura organizacional, que foi adotada para o presente estudo, considera que a cultura é definida como um atributo possuído pelas organizações, podendo os pesquisadores e gestores identificar, mudar e medi-lo empiricamente, principalmente por métodos quantitativos. Além disso, esta perspectiva considera que a cultura organizacional é um potencial preditor de resultados organizacionais (tal como a eficácia), tendo como interesse subjacente a busca de previsibilidade, de controle organizacional.

Em consonância com esta vertente epistemológica, foi escolhido o modelo denominado de Competing Value Model (CVM) para a análise da cultura organizacional, no presente estudo. Ao relacionar o enfoque organizacional ao ambiente interno ou externo e à estrutura organizacional, visando controle e estabilidade ou flexibilidade e mudança, emergem quatro tipos culturais: Cultura Clã ou Grupal; Cultura Hierárquica; Cultura de Adhocracia ou Inovativa; e Cultura de Mercado ou Racional. A representação gráfica das tipologias culturais é apresentada na Figura 1.

A Cultura Clã ou Grupal enfatiza valores como coesão, moral, participação e abertura. As organizações que possuem este tipo de cultura costumam incentivar o trabalho em grupo, o programa de envolvimento dos colaboradores e o comprometimento corporativo com os empregados. Assim, os indivíduos apresentam-se altamente comprometidos com a organização e o desenvolvimento deles se traduz em benefícios a longo prazo (CAMERON; QUINN, 2006).

A Cultura Hierárquica possui como principal aspecto um local de trabalho formal e estruturado, com regras e políticas que mantêm a organização integrada e enfatizam a estabilidade, eficiência e previsibilidade. Existem padrões e procedimentos que determinam as atividades das pessoas e mantêm um ritmo estável de atividades. A liderança se dá pela coordenação e organização (CAMERON; QUINN, 2006).

A Cultura de Adhocracia ou Inovativa direciona para mudanças e novos desafios, acreditando que o sucesso está na produção de serviços e produtos únicos e originais. O ambiente de trabalho é dinâmico, empreendedor e criativo. A liderança é visionária, inovativa e orientada para riscos. Ocorre a ênfase na atuação na fronteira do conhecimento, em produtos e/ou serviços e no crescimento rápido e aquisição de novos recursos. Ser o líder de um produto ou serviço é muito importante em culturas inovativas. A organização encoraja a liberdade e a iniciativa individual. O comprometimento com a experimentação e com a inovação é o que mantém a organização coesa (CAMERON; QUINN, 2006).

Já na Cultura de Mercado ou Racional, a organização é orientada para resultados. Ocorre a crença de que o ambiente externo não é benigno, e sim hostil, e que os consumidores realizam escolhas e estão interessados em valor. A principal tarefa da gerência é guiar a organização em direção à produtividade e ao alcance de metas e rendimentos. O principal negócio da organização é melhorar sua posição competitiva, assumindo que um propósito claro e uma estratégia agressiva levam a organização à produtividade e à rentabilidade (CAMERON; QUINN, 2006). 


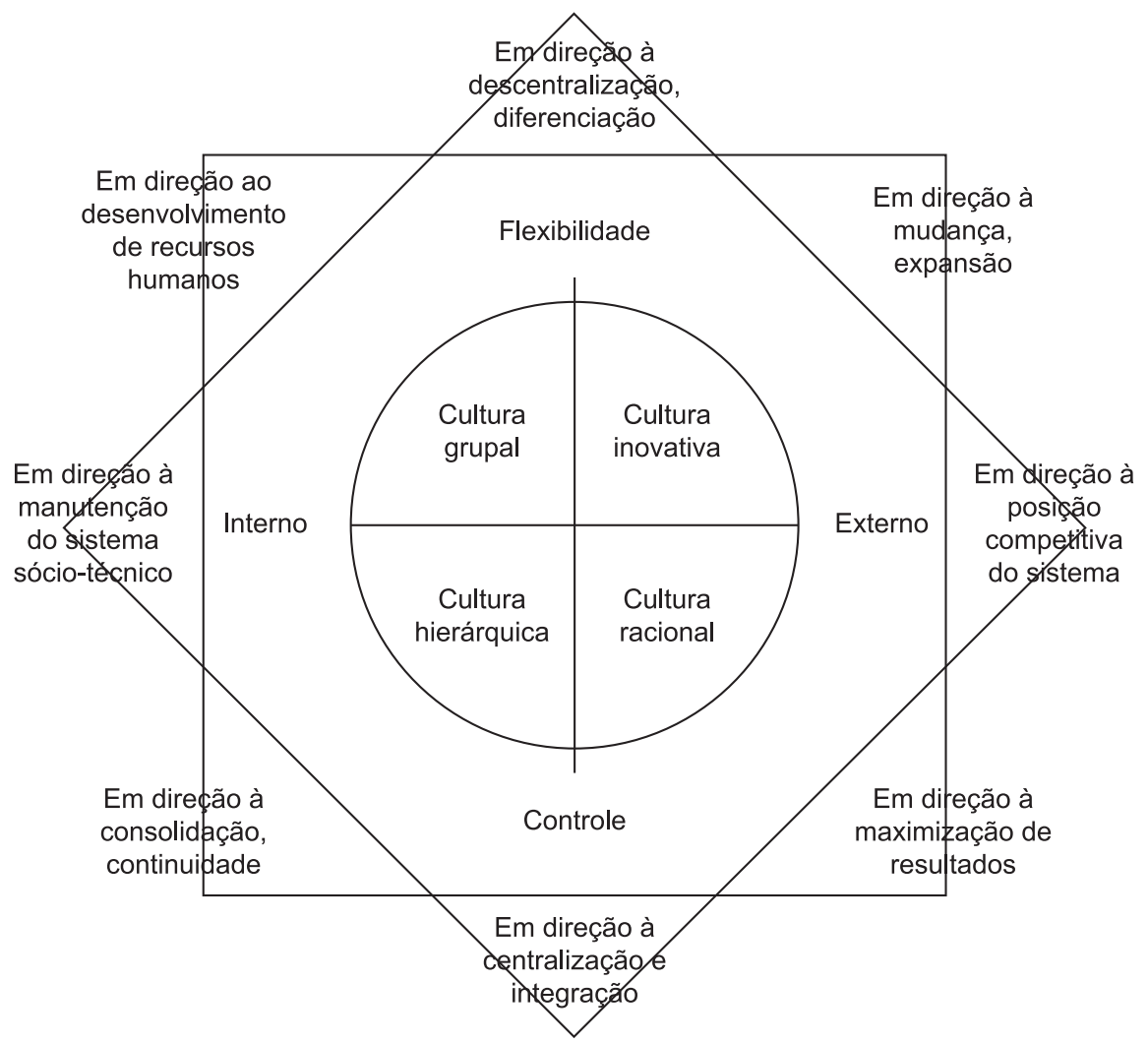

Figura 1. Principais dimensões do Competing Value Model e os tipos de Cultura Organizacional. Fonte: Santos (1998, 2000).

O CVM preconiza a coexistência das tipologias culturais nas organizações, enfatizando que é de suma importância o equilíbrio entre culturas, pois a ênfase em determinada cultura pode tornar a organização disfuncional (CAMERON; QUINN, 2006). O modelo também revela a importância da força cultural, que pode ser definida como a extensão em que os valores e crenças que permeiam a organização são ampla e intensamente compartilhados pelos seus membros (SANTOS, 1998), e estima que a cultura forte é aquela que mais incorpora as características de todos os quatro tipos culturais, possuindo médias elevadas em todas as tipologias a serem mensuradas.

\section{Clima organizacional}

O termo clima organizacional, assim como outros termos relacionados à ciência administrativa, tem origem em outras disciplinas. Neste caso, o termo advém da meteorologia, configurando-se em uma “analogia meteorológica” (KATZ; KAHN, 1975, p. 86).

Segundo Sá Leitão, Guimarães e Rosal (1998), existem, na área do comportamento organizacional, alguns assuntos que são de difícil compreensão e que se confundem em sua abrangência, sendo o clima organizacional um dos mais confusos e malcompreendidos.

A própria metáfora de onde se origina o termo clima denota a dificuldade em se precisar sua ocorrência e compreensão, quando se observa que as previsões do clima atmosférico dificilmente são precisas e seguras [...] (SÁ LEITÃO; GUIMARÃES; ROSAL, 1998, p. 2).

O termo clima organizacional é bastante difuso, inclusive, quando se analisa seu surgimento como ramo de interesse da pesquisa em administração. Isto ocorre porque vários pesquisadores do comportamento humano nas organizações já tratavam do tema, mesmo que de maneira implícita, desde os anos 30 do século XX.

O conceito é polêmico também por que muitos autores, a exemplo de Katz e Kahn (1975), o tratam como sinônimo de cultura, isto é, não existe consenso sobre definição de clima organizacional, apenas semelhanças de ideias, apesar do grande tempo decorrido desde o início das pesquisas nessa área. Oliveira (1995) argumenta que a expressão clima é relativamente nova, uma vez que, nos primórdios da pesquisa sobre o tema, usavam-se as expressões "atmosfera", "ambiente" ou "meio ambiente" e isso 
pode ter colaborado para essa falta de consenso. No entendimento de Santos (1999, p. 27):

O conceito de clima organizacional mostra um dilema em comum no esforço de descrever e compreender o comportamento humano. $\mathrm{O}$ problema é, em essência, o de operacionalizar o conceito para, então, medi-lo de forma confiável e válida. O que se evidencia, através dos trabalhos e pesquisas na área, é que existe um quase perfeito acordo entre os autores de que o clima organizacional é um conceito significativo, o qual tem importantes implicações para entender o comportamento humano nas organizações.

Assim, a comparação entre os conceitos de diversos autores se faz necessário para a identificação dos principais fatores envolvidos na análise do clima organizacional.

Forehand e Gilmer (1964, p. 362) definem clima organizacional como:

[...] o conjunto de características que descrevem uma organização e que: a) distingue uma organização da outra; b) mantém-se de certa forma permanente; e c) influencia o comportamento dos indivíduos na organização.

James e Jones (1974) propõem uma reconceituação do termo clima organizacional no sentido de diferenciar clima como um atributo individual e clima como um atributo organizacional. Ao se referir ao clima como um atributo individual, os autores defendem que deve ser empregada uma terminologia diferenciada, tal como "clima psicológico".

Para Martins et al. (2004) e Martins (2008), o clima organizacional está relacionado às percepções e interpretações comuns das dimensões das atividades, do ambiente e das políticas que caracterizam a organização.

Segundo Coda (1997), o clima organizacional reflete a tendência ou inclinação a respeito de até que ponto as necessidades da organização e das pessoas que dela fazem parte estão sendo efetivamente atendidas, sendo esse aspecto um dos indicadores da eficácia organizacional.

Para Rizzatti (2002), o estudo do clima organizacional, seja por meio do estudo das percepções ou das aspirações dos indivíduos, é um diagnóstico adequado da instituição, dispondo o pesquisador de duas alternativas básicas para a realização de um estudo envolvendo tal construto: na primeira, ele adota um conjunto específico de fatores ou categorias já conhecidos e consagrados na literatura, ou elabora o seu próprio modelo.

Neste trabalho, será utilizada a Escala de Clima Organizacional (ECO) apresentada por Martins et al. (2004) e Martins (2008). Optou-se por este instrumento pelo fato deste ser um instrumento relativamente novo, carecendo de estudos que reforcem sua aplicabilidade e, também, porque este foi desenvolvido no Brasil por pesquisadores brasileiros e, por isso, tem maior tendência de adaptação para aplicação na realidade do presente estudo.

\section{Diferenças e relações entre os construtos cultura e clima organizacionais}

Com o intuito de diferenciar os construtos cultura e clima organizacional, recorre-se inicialmente a Puente-Palacios (2002). A autora argumenta que a semelhança entre os dois conceitos pode ser justificada pela compreensão de que são eles dois elementos de um mesmo cenário, a organização, mas não são o mesmo fenômeno. Enquanto a cultura refere-se a um conjunto de normas, crenças, valores e mitos mais ou menos estável, que define os padrões de comportamento esperado dos membros, que a ela pertencem, e são utilizados como modelos de comparação e julgamento, tendo sua importância pelo fato de nortear o comportamento e o desempenho dos seus membros, o clima refere-se às representações compartilhadas das percepções dos membros sobre o agir da organização, construídas com base na cultura e de estabilidade menor, podendo mudar ao longo do tempo, mas, permanecendo o suficiente para serem significativas aos membros da organização, pois não são características passageiras ou de estado de ânimo da organização, mas padrões de ação e reação. Entretanto, essa distinção nem sempre apareceu de forma clara, havendo evidências de que os dois termos têm sido utilizados como sinônimos (BARKER, 1994 apud WALLACE; HUNT; RICHARDS, 1999, p. 551). Podemos citar como exemplo Katz e Kahn (1975), que, ao descreverem a estrutura sistêmica das organizações, citam clima e cultura como sinônimos.

Wallace, Hunt e Richards (1999) argumentam, utilizando os trabalhos de Schneider (1985) e de Ryder e Southey (1990 apud WALLACE; HUNT; RICHARDS, 1999, p. 551), que existe um relacionamento próximo, e algumas vezes ambíguo, entre cultura e clima organizacional que tem sido frequentemente negligenciado. Baseando-se em Field e Ableson (1982 apud WALLACE; HUNT; RICHARDS, 1999, p. 551), os autores citam ainda que, apesar do grande número de estudos sobre clima, as tentativas de definir o construto de maneira que o diferencie da cultura têm-se mostrado problemáticas.

Buscando a compreensão da diferença entre esses conceitos, apresentam-se, a seguir, as categorias constitutivas de cada um, segundo Verbeke, Volgering e Hessels (1998). Esses autores analisaram a evolução conceitual das definições de clima organizacional (32 definições) e cultura organizacional (54 definições) a partir de 1964 e 1979, respectivamente. Cada 
definição foi analisada por uma comissão de peritos, cuja tarefa foi identificar as categorias constitutivas dos conceitos. Foram encontradas como categorias comuns aos dois construtos: organização, membros, comportamento, compartilhar, conjunto e práticas. Estas categorias comuns a ambos os conceitos demonstram que há espaço compartilhado entre eles, enquanto as excludentes revelam que não existe completa superposição entre ambos, o que leva a crer que ambos podem ser usados simultaneamente, sem medo de estar sendo redundante com relação aos fenômenos. Puente-Palacios (2002) questiona até que ponto essas categorias são suficientemente amplas para abarcar a totalidade do conceito e, ao mesmo tempo, suficientemente estreitas para diferenciá-lo de outros. A autora cita (PUENTE-PALACIOS, 2002, p. 99):

A esse respeito cabe mencionar que o clima não é uma característica estática, mas dinâmica, do âmbito organizacional, por isso a sua estrutura não está apenas definida pela presença-ausência de determinados elementos, mas também pelo tipo de relação estabelecida entre eles. Assim, ainda que o clima seja composto por categorias conceituais comuns a outros eventos, a forma como elas se organizam e se relacionam constitui o elemento diferencial que estabelece a sua particularidade.

Também com a intenção de diferenciar esses dois construtos, Cameron e Quinn (2006) citam que o clima em comparação com a cultura se refere a atitudes, sentimentos e percepções mais temporárias por parte dos indivíduos. Citam, ainda, que a cultura organizacional é um atributo central e de lenta mudança das organizações, enquanto o clima, por se basear em atitudes, pode mudar mais rápida e dramaticamente. A cultura se refere a algo implícito, a aspectos frequentemente indiscerníveis das organizações, enquanto o clima se refere a atributos mais públicos, observáveis das organizações. A cultura inclui valores centrais e interpretações consensuais sobre como as coisas são, já o clima inclui perspectivas individualistas que são modificadas frequentemente de acordo com a mudança das situações e o surgimento de novas informações.

Neste ponto, há que se destacar a divergência existente entre o que defendem Cameron e Quinn (2006) e o que defende Puente-Palacios (2002) a respeito das características do clima organizacional. Apesar dos autores concordarem a respeito da menor estabilidade do clima organizacional quando comparado à cultura organizacional, Cameron e Quinn (2006) afirmam que essa mudança pode ocorrer rápida e frequentemente, estando ligada a perspectivas individualistas, enquanto Puente-Palacios (2002) defende que essa mudança não envolve características passageiras e sim, padrões de ação e reação. Enquanto Puente-Palacios (2002) considera as percepções coletivas dos membros da organização, o que gera esses padrões de ação e reação, Cameron e Quinn (2006) consideram as percepções individuais que são mais flexíveis. Essa diferença no ponto de vista dos autores não invalida suas afirmações, principalmente por que eles concordam no ponto fulcral a respeito do clima organizacional, que é a percepção dos indivíduos, seja individual ou coletivamente, a respeito das mais diversas características organizacionais.

Wallace, Hunt e Richards (1999) citam que a maior distinção entre os dois construtos reside no fato de o clima de uma organização consistir de percepções compartilhadas, enquanto a cultura de uma organização é feita de pressupostos compartilhados.

Já para Souza (1978, p. 38):

O clima é uma resultante das variáveis culturais. Quando estas são alteradas, ocasionam alterações no clima. Curiosamente, o clima é mais perceptível do que suas fontes causais. É comparável a um perfume: percebe-se o efeito, sem conhecer os ingredientes, embora, às vezes seja possível identificar alguns deles.

Percebe-se, no discurso de Souza (1978), que há o reconhecimento da relação entre cultura e clima organizacional, em que a cultura influencia decisivamente o clima. Outros autores concordam com Souza (1978), entre eles, Tamayo (1999), Martins et al. (2004) e Martins (2008), Santos (2000), Schneider (1985) e Wallace, Hunt e Richards (1999), sendo que estes últimos encontraram, inclusive, indícios empíricos da relação entre cultura e clima organizacional em seu trabalho.

Porém, existem diversos autores que não reconhecem esta relação. Savoie e Brunet (2000), após exaustivo resgate teórico acerca dos referidos temas, negam a relação entre cultura e clima organizacional, argumentando que os construtos vêm de diferentes tradições científicas, são conceitualmente diferentes, são produtos de diferentes determinantes e dão origem a diferentes efeitos organizacionais. Ainda citam os trabalhos de Chagnon (1991 apud SAVOIE; BRUNET, 2000, p. 192) e do Grupo de Pesquisa Sobre o Comportamento Antissocial, do Departamento de Psicologia da Universidade de Montreal (GRC-A, 2000 apud SAVOIE; BRUNET, 2000, p. 192), que encontraram índices de correlação muito baixos entre os construtos clima e cultura organizacional. Mais recentemente, tem-se o trabalho de Sá et al. (2006), que também não encontraram correlação empírica entre cultura e clima.

Percebe-se, assim, que existe, mesmo depois de anos de pesquisa, bastante contradição entre os autores com relação à existência de relação entre cultura e 
clima organizacionais, o que evidencia a necessidade da continuidade dos estudos nessa área.

\section{Procedimentos metodológicos}

A presente pesquisa pode ser caracterizada como exploratória, pois buscou familiarizar-se com o fenômeno da cultura e do clima organizacionais no ambiente das indústrias de laticínios do Rio Grande do Norte, aumentar a gama de conhecimento sobre o objeto proposto e possibilitar o surgimento de novas ideias a serem aprofundadas em trabalhos futuros (GIL, 2009). O presente estudo também é descritivo, pois analisou os dados buscando suas relações. De acordo com Gil (2009, p. 42), um estudo descritivo tem por "[...] objetivo primordial a descrição das características de determinada população ou fenômeno ou, então, o estabelecimento de relações entre variáveis [...]". Ainda, este estudo é conclusivo-causal (MALHOTRA, 2001), pois tem como objetivo a descrição das características de determinada população e o estabelecimento de relações de causa e efeito entre variáveis, utilizando técnicas padronizadas de coleta de dados. Possui objetivos bem definidos, procedimentos formais e bem estruturados e dirigidos para a solução de problemas ou a avaliação de alternativas de cursos de ação. Além disso, os dados obtidos estão sujeitos à análise quantitativa (MALHOTRA, 2001), que foi o método adotado pelo presente estudo.

A escolha por uma metodologia quantitativa deveu-se ao fato de que, como apresentado anteriormente, o viés funcionalista adotado pelo presente estudo para análise de clima e cultura organizacionais e o seu objetivo geral exigem o emprego deste método. Assim, este estudo tem a intenção de garantir com precisão os resultados, evitar distorções de análise e interpretação (RICHARDSON, 2008). Vale salientar que, neste estudo, não houve criação de categorias e sim aplicação de categorias anteriormente criadas.

A população foi definida, inicialmente, pelo cadastro da Federação das Indústrias do Estado do Rio Grande do Norte (FIERN). Existiam, no momento do estudo, 25 indústrias cadastradas junto a este órgão, classificadas como Indústrias de Laticínios. Essas Indústrias estavam distribuídas em 22 diferentes cidades do Rio Grande do Norte, totalizando 825 funcionários. Apesar do grande número de indústrias, estas eram bastante homogêneas com relação às suas características, sendo todas classificadas como pequenas empresas, tendo seu principal mercado consumidor no próprio Estado do Rio Grande do Norte, sendo constituídas por maioria de homens com baixa escolaridade. Dos 825 funcionários deste setor, 211 participaram do presente estudo $(25,6 \%)$. Não houve qualquer restrição quanto à escolaridade, pois a aplicação dos questionários foi acompanhada pelos pesquisadores, e todas as dúvidas foram esclarecidas no momento em que surgiram. É importante ressaltar que a pesquisa ocorreu com funcionários dos mais diversos níveis hierárquicos, com o intuito de evitar aquilo que Barbosa (2003) chama de cultura corporativa ou cultura da cúpula, diferenciando-a da verdadeira cultura organizacional presente nas organizações. Os questionários foram aplicados entre novembro de 2009 e fevereiro de 2010.

Já a estruturação e montagem final do instrumento de coleta de dados foram divididas em três partes. A primeira delas consistiu em informações que abrangem aspectos relacionados aos respondentes como: sexo, idade, estado civil, anos de empresa, cargo ocupado e escolaridade. A segunda constou de um conjunto de 24 proposições, capazes de gerar quatro perfis que representam diferentes padrões culturais da organização (Cultura Clã, Cultura Inovativa, Cultura de Mercado e Cultura Hierárquica), conhecidos como Competing Value Model (CVM) ou Tipologia de Quinn (QUINN; ROHRBAUGH, 1981; CAMERON; QUINN, 2006). O instrumento, originalmente em inglês, foi utilizado segundo a tradução de Santos (2000). Este instrumento é, também, comumente utilizado para se aferir a tipologia considerada ideal, porém, no presente trabalho não haverá questionamentos acerca desta, por não se tratar do foco da pesquisa. A terceira e última parte do questionário apresentou variáveis de mensuração do clima organizacional, segundo Martins et al. (2004) e Martins (2008). A escala de medida de clima organizacional (ECO) compõe-se originalmente por 63 itens, divididos em 5 fatores: "Apoio da chefia e da organização" refere-se ao suporte afetivo, estrutural e operacional da chefia e da organização fornecido aos empregados no desempenho diário de suas atividades no trabalho, sendo composto por 21 variáveis; "Recompensa" sugere que diversas formas de recompensa são utilizadas pela empresa para premiar a qualidade, a produtividade, o esforço e o desempenho do trabalhador, sendo composto por 13 variáveis; "Conforto físico" diz respeito ao ambiente físico, à segurança e ao conforto proporcionados pela empresa aos empregados, sendo composto por 13 variáveis; "Controle/Pressão", composto por 9 variáveis, refere-se ao controle e pressão exercidos pela empresa e pelos supervisores sobre o comportamento e desempenho dos empregados; e "Coesão entre colegas" exalta a união, vínculos e colaboração entre os colegas de trabalho, sendo formado por 7 variáveis.

Para o presente estudo, utilizou-se este modelo, porém, com modificações, de modo que foram utilizadas as 3 variáveis que apresentavam maiores escores fatoriais em cada fator, segundo o descrito por Martins et al. (2004). Desta maneira, obteve-se um instrumento "enxuto" para análise do clima 
organizacional, contendo 15 variáveis divididas em 5 fatores.

$\mathrm{O}$ instrumento completo, estruturado e autopreenchível, foi respondido por uma escala de concordância, do tipo Likert, de 5 pontos, sendo que o valor 1 correspondia a "discordo totalmente" e o 5 a "concordo totalmente", com exceção da primeira parte relativa à caracterização dos respondentes que foi respondida livremente.

É importante salientar que, de todos os fatores utilizados neste estudo, tanto para cultura quanto para clima, somente o fator de análise do clima organizacional "controle/pressão" tem "sentido negativo". O argumento da autora do instrumento é que, no caso deste fator, "quanto maior o resultado, pior será o clima porque maior será o controle e a pressão exercidos sobre os empregados" (MARTINS, 2008, p. 34). Porém, segundo Hofstede (1982, 1997), o Brasil é um país com elevado "índice de controle da incerteza" e elevado "índice de distância hierárquica". Para o autor, os membros de uma sociedade de elevado "índice de controle da incerteza" sentem-se cômodos em meios estruturados, deixando-se o menor espaço possível ao acaso. Acerca do elevado "índice de distância hierárquica", o autor cita que nesses contextos, superiores e subordinados, consideram-se desiguais por natureza, tendendo as organizações a maximizarem a centralização do poder num grupo restrito de pessoas. Quando se tem uma elevada "distância hierárquica" e um elevado "controle da incerteza" o exercício discricionário por parte dos superiores, substitui, até certo ponto, a necessidade emocional de normas internas. Diante do exposto, tem-se que, para o estudo em questão, o fator "controle/ pressão" pode não ter um "sentido negativo" e sim positivo. Com isso, optou-se por manter os valores originais atribuídos pelos respondentes às variáveis pertencentes a este fator ao se analisar estatisticamente os dados.

\section{Resultados e discussão}

Aos se analisar os 211 questionários respondidos pelos funcionários das indústrias de laticínios pesquisadas, foram obtidos os seguintes valores para as estatísticas descritivas das variáveis de identificação dos respondentes: $87,2 \%$ são homens e $12,8 \%$ são mulheres; com média de 37 anos de idade; $67,8 \%$ são casados e $32,2 \%$ são solteiros; com média de 6,7 anos de empresa; são $73,9 \%$ operários, $10 \%$ encarregados, $6,6 \%$ auxiliares administrativos, $3,8 \%$ vendedores e $5,7 \%$ gerentes; $35,1 \%$ possuem $1^{\circ}$ grau incompleto, $13,3 \%$ possuem $1^{\circ}$ grau completo, $8,0 \%$ possuem $2^{\circ}$ grau incompleto, $29,4 \%$ possuem $2^{\circ}$ grau completo, $4,7 \%$ possuem $3^{\circ}$ grau incompleto, $7,6 \%$ possuem $3^{\circ}$ grau completo e $1,9 \%$ possuem pós-graduação completa.
Com o intuito de verificar como o instrumento se comportou, realizou-se o teste de confiabilidade. Os valores para os coeficientes $\alpha$ de Cronbach são apresentados na Tabela 1.

Os resultados para o teste de consistência interna, ou teste de confiabilidade, atestaram a viabilidade da utilização do referido instrumento para a realidade pesquisada, uma vez que todos os fatores obtiveram valores iguais ou superiores a 0,70 , sendo este o valor mínimo ideal (HAIR JUNIOR et al., 2007). Ao se comparar com outros autores brasileiros, tem-se que Santos (2000) encontrou 0,83 para "cultura clã", 0,83 para "cultura inovativa", 0,80 para "cultura hierárquica" e 0,78 para "cultura de mercado", enquanto Martins et al. (2004) e Martins (2008) encontrou 0,92 para "apoio da chefia e da organização", 0,88 para "recompensa", 0,86 para "conforto físico", 0,78 para "controle/pressão" e 0,78 para "coesão entre colegas".

Tabela 1. Coeficientes $\alpha$ de Cronbach.

\begin{tabular}{lcc}
\hline \multicolumn{1}{c}{ Fatores } & $\begin{array}{c}\text { Número de } \\
\text { variáveis }\end{array}$ & $\begin{array}{c}\alpha \text { de } \\
\text { Cronbach }\end{array}$ \\
\hline Cultura clã & 6 & 0,78 \\
Cultura inovativa & 6 & 0,75 \\
Cultura de mercado & 6 & 0,78 \\
Cultura hierárquica & 6 & 0,72 \\
Apoio da chefia & 3 & 0,78 \\
Recompensa & 3 & 0,86 \\
Conforto físico & 3 & 0,76 \\
Controle/pressão & 3 & 0,75 \\
Coesão entre colegas & 3 & 0,80 \\
\hline
\end{tabular}

Fonte: Dados da pesquisa (2010).

Tabela 2. Resultados para a Cultura Organizacional.

\begin{tabular}{lrc}
\hline \multicolumn{1}{c}{ Itens } & Média & Desvio padrão \\
\hline Cultura clã & 3,6509 & 0,7930 \\
Cultura inovativa & 3,5008 & 0,7919 \\
Cultura de mercado & 3,5569 & 0,7780 \\
Cultura hierárquica & 3,5047 & 0,7257 \\
Força cultural & 14,2133 & 2,6881 \\
\hline
\end{tabular}

Fonte: Dados da pesquisa (2010).

Tabela 3. Resultados para o Clima Organizacional.

\begin{tabular}{lcc}
\hline \multicolumn{1}{c}{ Itens } & Média & Desvio padrão \\
\hline Apoio da chefia & 3,8420 & 0,9254 \\
Recompensa & 3,2227 & 1,1549 \\
Conforto físico & 3,9226 & 0,8164 \\
Controle/pressão & 3,5893 & 1,0167 \\
Coesão entre colegas & 3,7093 & 0,9544 \\
Média clima & 3,6572 & 0,4837 \\
\hline
\end{tabular}

Fonte: Dados da pesquisa (2010). 
Com o intuito de evidenciar as tipologias e a força cultural, e os resultados dos fatores do clima organizacional e sua média final, foram elaboradas as Tabelas 2 e 3.

Com esses resultados preliminares, percebe-se que a tipologia cultural predominante nas organizações pesquisadas foi a cultura clã, enquanto que a média do clima, segundo Martins (2008), evidencia um clima bom nessas organizações (valores próximos a 4). Nestas organizações, as pessoas tendem a ser vistas como membros de um sistema social comum, com um forte senso de coesão. Enfatizam-se o desenvolvimento de recursos humanos, bem como o seu comprometimento, o incentivo à formação de equipes de trabalho e de cooperação mútua.

Posteriormente, foi então realizada a análise de Clusters com o intuito de reunir os respondentes com perfis culturais semelhantes em grupos considerados distintos. A Tabela 4 mostra o resultado da análise de Clusters, apresentando a média e o desvio padrão, tanto para a força cultural como para o clima, dos dois grupos formados.

Com base na Tabela 4, nota-se que o primeiro grupo, com 117 indivíduos, apresenta força cultural menor que o segundo grupo, sendo, por isso, denominado grupo de cultura "fraca". Consequentemente, o segundo grupo, com 94 indivíduos, foi denominado grupo de cultura "forte". Para verificar se a cultura realmente discriminava os dois grupos, realizou-se a análise discriminante, que obteve $97,2 \%$ de acerto, comprovando o sucesso da análise de Clusters. Ao se analisar, na mesma tabela, os valores do clima organizacional para os dois grupos, evidencia-se que o grupo de cultura "forte" apresenta valores substancialmente maiores. Entretanto, as diferenças, tanto para cultura quanto para clima, não são suficientes para garantir sua representatividade. Para isto, o teste F-ANOVA foi realizado, tanto para os valores da cultura quanto para os valores de clima, testando a hipótese nula de que a média das duas populações é igual. Com base nos resultados do Teste F-ANOVA, rejeita-se a hipótese nula de que as médias dos grupos 1 e 2, para os valores de cultura e clima, são iguais (sig. = 0,000). Este resultado permite afirmar que as diferenças entre os valores para forças culturais e para o clima são estatisticamente significantes, evidenciando que o grupo de cultura "forte" tem melhor percepção sobre o clima da organização. Este resultado empírico reforça o defendido por vários outros autores, como Puente-Palacios (2002), Souza (1978), Schneider (1985), Cameron e Quinn (2006), Martins et al. (2004), Martins (2008), Wallace, Hunt e Richards (1999), Santos (2000) e Tamayo (1999). De uma forma geral estes autores defendem que a cultura influencia diretamente o clima organizacional, sendo o clima expressão da própria cultura.

Esta evidência da influência da cultura sobre o clima organizacional das indústrias pesquisadas ainda encontra respaldo no próprio modelo cultural, o CVM, uma vez que este defende que uma cultura forte está associada com a homogeneidade de esforços, clareza de foco e unicidade organizacional (CAMERON; QUINN, 2006), o que pode promover um ambiente amistoso dentro da organização.

Por fim, fez-se a análise de correlação canônica entre os fatores de cultura e clima organizacionais. Utilizando-se dos indícios empíricos preliminares de que uma cultura "forte" influencia positivamente o clima organizacional, procurou-se saber a relação entre os perfis culturais e os fatores constituintes do clima organizacional. Fez-se a análise considerando os fatores de cultura organizacional como variáveis independentes $\left(\mathrm{X}_{\mathrm{i}}\right)$ e os fatores de clima organizacional como variáveis dependentes $\left(\mathrm{Y}_{\mathrm{i}}\right)$. Assim, o conjunto preditor ou explicativo será formado por "Cultura Clã", "Cultura Inovativa", "Cultura de Mercado" e

Tabela 4. Média dos grupos formados pela Análise de Clusters.

\begin{tabular}{cccccc}
\hline \multirow{2}{*}{ Grupos } & \multirow{2}{*}{ Indivíduos } & \multicolumn{2}{c}{ Média } & \multicolumn{2}{c}{ Desvio padrão } \\
\cline { 3 - 6 } & & Cultura & Clima & Cultura & Clima \\
\hline 1 & 117 & 12,268 & 3,237 & 1,642 & 0,506 \\
2 & 94 & 16,635 & 4,180 & 1,505 & 0,462 \\
\hline
\end{tabular}

Fonte: Dados da pesquisa (2010).

Tabela 5. Correlação canônica e teste de significância.

\begin{tabular}{cccrrrc}
\hline $\begin{array}{c}\text { Função } \\
\text { canônica }\end{array}$ & $\begin{array}{c}\text { Correlação } \\
\text { canônica }(\mathbf{R})\end{array}$ & $\mathbf{R}^{2}$ & Quiquadrado & GL & Sig. & $\lambda$ de Wilks \\
\hline 1 & 0,7886 & 0,6219 & 259,3246 & 20,0000 & 0,0000 & 0,2822 \\
2 & 0,3841 & 0,1476 & 59,9225 & 12,0000 & 0,0000 & 0,7465 \\
3 & 0,3478 & 0,1209 & 27,1939 & 6,0000 & 0,0001 & 0,8758 \\
4 & 0,0611 & 0,0037 & 0,7676 & 2,0000 & 0,6813 & 0,9963 \\
\hline
\end{tabular}

Fonte: Dados da pesquisa (2010). 
"Cultura Hierárquica", enquanto o conjunto critério ou explicado, será formado por "Apoio da chefia e da organização", "Recompensa", "Conforto físico", "Controle/Pressão" e "Coesão entre colegas". A Tabela 5 apresenta os primeiros resultados para a correlação canônica.

Para decidir qual função canônica analisar, são necessários três critérios. Segundo Hair Junior et al. (2007, p. 367),

[...] os três critérios são (1) nível de significância estatística da função, (2) magnitude da correlação canônica e (3) medida de redundância para o percentual de variância explicada a partir dos dois conjuntos de dados (HAIR JUNIOR et al. 2007, p. 367),

Com base na Tabela 5, pode-se afirmar que a função 1 apresenta índice de correlação canônica substancialmente maior que as outras, o que a leva a ter maior raiz canônica, apesar de outras duas funções terem apresentado significância estatística ao nível de $1 \%$. Com isso, a função 1 foi a escolhida para ser analisada neste estudo.

A correlação canônica mede a força da associação entre os dois conjuntos de variáveis. A força do relacionamento entre os pares de variáveis é refletida principalmente pelo índice de correlação canônica da função escolhida. $\mathrm{O}$ valor de 0,7886 indica forte correlação positiva e significativa. Quando elevado ao quadrado, tal coeficiente representa a quantidade de variância em um composto linear da função canônica atribuída a outro composto da mesma função. Sendo assim, pode-se dizer que, $62,19 \%$ da variação em um composto linear da primeira função são influenciados pela variação no outro composto que a compõe.

Por fim, para identificar o tipo de relação entre os fatores de cultura e clima organizacionais, foram analisados os resultados das cargas e dos pesos canônicos.
Os "pesos canônicos" são similares aos "betas" de uma regressão múltipla. Entretanto, o uso dos pesos para analisar uma função canônica pode acarretar alguns problemas como instabilidade dos valores em função da amostra e dificuldades de interpretação dos valores em um ambiente com multicolinearidade. Isso nos leva a analisar a carga canônica para determinar a importância relativa das variáveis para cada composto canônico. Os altos valores das cargas canônicas, gerados para a maior parte das variáveis, reforça a viabilidade do modelo.

Com base na Tabela 6, verifica-se que todos os perfis culturais influenciam fortemente e positivamente os fatores de clima organizacional, se configurando como fortes preditores deste. É importante destacar que o fator de clima organizacional "controle/pressão" foi influenciado positivamente por todos os perfis culturais, comprovando que, nas organizações estudadas, o controle e a pressão exercidos pela organização são vistos como benéficos para o clima, atestando a viabilidade da modificação realizada com relação à interpretação dos resultados obtidos pelo uso da escala elaborada por Martins et al. (2004) e Martins (2008).

Nas organizações analisadas, as tipologias que mais influenciam os fatores de clima organizacional são a cultura clã e a cultura inovativa. Isto talvez se deva ao fato de que a cultura clã incentiva o trabalho em grupo, os programas de envolvimento dos funcionários e o comprometimento corporativo com os empregados, além de enfatizar valores como coesão, moral, participação e abertura, criando uma relação quase paterna da organização com o funcionário, gerando segurança. Da mesma forma, a cultura inovativa incentiva o trabalho dinâmico, empreendedor e criativo, encorajando a liberdade e iniciativa individual, promovendo um ambiente de trabalho em que os erros são tolerados, sendo a liderança visionária e orientada para riscos. Neste ponto, é interessante citar Hofstede (1997), que faz uma importante diferenciação entre risco e incerteza.

Tabela 6. Cargas e Pesos Canônicos.

\begin{tabular}{lcc}
\hline \multicolumn{1}{c}{ Variáveis Dependentes } & Cargas canônicas & Pesos canônicos \\
\hline Y $_{1}$ Apoio da chefia e da organização & 0,7458 & 0,2535 \\
Y $_{2}$ Recompensa & 0,7911 & 0,3749 \\
Y $_{3}$ Conforto físico & 0,3729 & 0,0397 \\
Y $_{4}$ Controle/Pressão & 0,7965 & 0,4611 \\
Y $_{5}$ Coesão entre colegas & 0,6010 & 0,2201 \\
\hline Independentes & & 0,3012 \\
X $_{1}$ Cultura clã & 0,8801 & 0,3766 \\
$X_{2}$ Cultura inovativa & 0,9092 & 0,1819 \\
$X_{3}$ Cultura de mercado & 0,8196 & 0,2827 \\
$X_{4}$ Cultura hierárquica & 0,8609 & \\
\hline
\end{tabular}

Fonte: Dados da pesquisa (2010). 
$\mathrm{O}$ autor cita que o risco centra-se em algo concreto enquanto a incerteza não tem objeto e não está ligada a nenhuma probabilidade.

Logo que a incerteza se exprima em termos de risco, deixa de ser uma fonte de ansiedade. Ela pode transformar-se em uma fonte de medo, mas pode também ser aceita como fazendo parte da rotina diária, como o risco de conduzir um automóvel ou praticar um esporte (HOFSTEDE, 1997, p. 139).

Desta forma, não há contradição no fato de haver incentivo a assumir riscos, nas organizações pesquisadas, e elas estarem inseridas em uma sociedade com elevado nível de "controle da incerteza".

É importante destacar que as tipologias culturais clã e inovativa estão inseridas na dimensão "flexibilidade" do modelo de Quinn, ou seja, direcionadas para a descentralização, o que pode ser um indicativo de que, nas organizações estudadas, os respondentes teriam melhor percepção do clima organizacional se tivessem maior autonomia em suas atividades. Este fato gera contradição, uma vez que os fatores do clima organizacional que mais foram afetados pelos perfis culturais foram "Recompensa" e "controle/ pressão", fatores esses ligados à produtividade, ao incentivo à qualidade, ao esforço e ao desempenho. As características desses fatores estão mais voltadas para a dimensão "controle" do modelo de Quinn. Pode-se discutir esse resultado novamente baseando-se em Hofstede (1982, 1997). Assumindo-se que o Brasil possui alto índice de "feminilidade", o fato das organizações pesquisadas apresentarem perfis culturais voltados para a dimensão "flexibilidade" acaba por estar coerente com a maior permissividade inerente a este tipo de sociedade. Este fato pode influenciar positivamente os fatores "recompensa" e "controle/ pressão", justamente por suavizá-los, tornando-os, antes de tudo, um instrumento para o atendimento da necessidade emocional por redução de incerteza, inerente a uma sociedade com elevado índice de "controle da incerteza".

Ao se fazer este tipo de recorte para análise, corre-se o risco de estar sendo reducionista, principalmente quando se analisa resultados que apresentam fortes relações em todas as "direções". Pode-se constatar, por exemplo, que os perfis culturais "clã" e "inovativa" também influenciam fortemente os fatores "apoio da chefia e da organização" e "coesão entre colegas", comprovando a existência de valores como coesão, moral, participação e abertura, ao mesmo tempo que encoraja a liberdade e a iniciativa individual. Da mesma maneira, os perfis culturais "mercado" e "hierárquica" influenciam fortemente os fatores do clima organizacional, sendo coerentes com a percepção acerca da "recompensa" e do "controle/pressão". Tem-se, ainda, que o fator que recebe menos influência dos perfis culturais é o "conforto físico", mesmo sendo este coerente com um perfil organizacional "clã" que é voltado para o desenvolvimento dos recursos humanos e para o cuidado com as pessoas.

Essa complexidade, que envolve as relações encontradas no presente estudo, está de acordo com o modelo cultural de Quinn, que focaliza os dilemas relativos ao sistema organizacional e, por isso, faz referência a valores que competem entre si. O modelo mostra que as organizações podem ser adaptáveis e flexíveis, tendo estabilidade e controle (SÁ et al., 2006). Outra comprovação do modelo cultural de Quinn, ao se analisar as indústrias pesquisadas, diz respeito ao equilíbrio como algo a ser perseguido pelas organizações, o que ficou evidente pela forma como todos os perfis culturais influenciaram fortemente $\mathrm{e}$ positivamente os fatores do clima organizacional. Tem-se que a ênfase em determinada cultura pode tornar a organização disfuncional (CAMERON; QUINN, 2006; SANTOS, 1998, 2000).

Por fim, para efeito de comparação, tem-se que Wallace, Hunt e Richards (1999), ao utilizarem a tipologia de Hofstede (1982) para análise da cultura organizacional, encontraram que os fatores "distância hierárquica" e "controle da incerteza" estavam positivamente correlacionados com o fator do clima organizacional "conflito e ambiguidade", e que o fator cultural "distância hierárquica" ainda estava correlacionado com o fator do clima "confiança mútua", comprovando a relação existente entre os construtos clima e cultura organizacionais. Coadunando a esta informação tem-se a conclusão de Souza (1978), defendendo que o equilíbrio entre os elementos culturais "preceitos" e "caráter" resulta em coesão, moral elevado, segurança e satisfação. De maneira antagônica, Sá et al. (2006), ao utilizarem o modelo CVM para medir os perfis culturais e o modelo de Kolb para medir o clima organizacional, não encontraram correlação entre os dois construtos, porém, não apresentaram qualquer teste estatístico para refutar tal ideia, o que é passível de questionamentos, principalmente quando trata-se de um estudo quantitativo. Savoie e Brunet (2000) também não reconhecem a relação entre cultura e clima organizacional, utilizando, para comprovar suas ideias, os estudos de Chagnon (1991 apud SAVOIE; BRUNET, 2000, p. 192) e do Grupo de Pesquisa Sobre o Comportamento Antissocial, do Departamento de Psicologia da Universidade de Montreal (GRC-A, 2000 apud SAVOIE; BRUNET, 2000, p. 192). Nestes estudos, foram apresentadas, respectivamente, correlações de 0,24 e 0,35 entre as medidas de cultura e clima organizacionais, valores estes bem inferiores aos encontrados para o presente estudo. 


\section{Considerações finais}

Depois da apresentação dos resultados do presente estudo, tem-se subsídios para realizar as considerações finais visando atender ao objetivo geral da pesquisa: investigar a existência, ou não, de relação entre a cultura organizacional e o clima organizacional, tendo, como locus de pesquisa, indústrias de laticínios do Rio Grande do Norte.

Em relação ao objetivo de identificar o tipo de perfil cultural das empresas estudadas, tem-se que todos os quatro perfis foram encontrados de forma equilibrada nas organizações pesquisadas, com ênfase para os perfis "cultura clã" e cultura "mercado". Enquanto o perfil "cultura clã" está voltado para o desenvolvimento dos recursos humanos, o perfil "cultura mercado" está voltado para a maximização dos resultados, ocupando dimensões opostas no CVM. Isto demonstra que as tipologias culturais não são autoexcludentes, podendo existir conjuntamente numa mesma organização.

Em relação ao objetivo de identificar o clima organizacional das organizações pesquisadas, observa-se que possuem um clima bom, baseando-se na classificação de Martins (2008), com destaque para os fatores "apoio da chefia e da organização" e "conforto físico", fatores estes bem coerentes com a tipologia "cultura clã".

No que concerne ao terceiro e último objetivo específico, utilizou-se a correlação canônica para medir o grau das relações entre os perfis culturais e os fatores do clima organizacional. Os resultados apontam para uma forte relação entre os perfis culturais e os fatores do clima organizacional, nos quais uma parcela substancial da variância das variáveis dependentes (fatores do clima organizacional) é explicada pelas variáveis independentes (perfis culturais). Pode-se perceber que houve forte influência positiva dos perfis culturais sobre os fatores do clima.

Por fim, os resultados nos permitem concluir que há relações entre os perfis culturais e os fatores determinantes do clima organizacional nas organizações pesquisadas. Essa constatação é consistente com o que é defendido por autores como Puente-Palacios (2002), Souza (1978), Schneider (1985), Cameron e Quinn (2006), Martins et al. (2004), Martins (2008), Wallace, Hunt e Richards (1999), Santos (2000) e Tamayo (1999), e vai de encontro ao que é defendido por autores como Savoie e Brunet (2000) e Sá et al. (2006). Há que se destacar que não se pode falar que somente a cultura influencia o clima das organizações pesquisadas, uma vez que fatores ambientais e pessoais também influenciam sua percepção, como bem apresentam Puente-Palacios (1995) e Johnston (1976). Além disso, autores como Souza (1978) citam a presença de uma "causalidade circular" entre esses dois construtos, de maneira que o clima também influencie a cultura das organizações.
Assim, as informações presentes neste estudo podem servir de base para que os gestores da área de recursos humanos possam melhor gerir o potencial de seu quadro de funcionários, visando unicidade de esforços na busca da tão almejada melhoria de performance organizacional. O estudo mostrou, de modo empírico, que a ênfase por parte dos gestores da área de recursos humanos em ações inerentes a um único perfil de cultura organizacional pode tornar a organização disfuncional, uma vez que todos os perfis culturais identificados influenciaram o clima organizacional. Por exemplo, podemos citar que o investimento no desenvolvimento da equipe de trabalho como única ação para promover a melhoria do clima organizacional, visando a melhoria de performance, pode não ser suficiente se não vier coordenada com outras ações inerentes a outros perfis culturais, como a busca por resultados, manutenção e continuidade de práticas positivas dentro da organização e foco na melhoria contínua.

Como recomendação para futuros estudos, tem-se a necessidade de analisar os diversos fatores que podem influenciar a relação entre cultura e clima organizacionais, buscando analisá-la de forma "macro", envolvendo outras características da organização e do ambiente em que esta se insere. Para desenvolver tal estudo, utilizar-se-ia a modelagem de equações estruturais que permite estimar múltiplas e interrelacionadas relações de dependência e representar conceitos não observados nessas relações, além de explicar o erro de mensuração no processo de estimação.

Além disso, tem-se, como necessidade urgente, a investigação da validade discriminante entre as medidas de cultura e clima organizacional, devido à proximidade conceitual entre esses dois construtos.

\section{Referências}

BARBOSA, L. Igualdade e Meritocracia. São Paulo: FGV, 2003.

CAMERON, K. S.; QUINN, R. E. Diagnosing and changing organizational culture. San Francisco: Jossey-Bass, 2006.

CODA, R. Pesquisa de clima organizacional e gestão estratégica de recursos humanos. In: BERGAMINI, C. W.; CODA, R. Psicodinâmica da vida organizacional: motivação e liderança. São Paulo: Atlas, 1997.

COSTA, G. V. Cultura e valores organizacionais Florianópolis: Insular, 1999.

DEAL, T.; KENNEDY, A. Corporate Culture: the rites and rituals of corporate life. Massachusets: Addison-Wesley, 1982.

FOREHAND, G. A.; GILMER, B. H. Environmental variation in studies of organizational behavior. Psychological Bulletin, v. 62, n. 6, Dec 1964. PMid:14242590. http://dx.doi.org/10.1037/h0045960

FREITAS, M. E. Cultura Organizacional: formação, tipologias e impacto. São Paulo: Makron Books, 1991. 
FREITAS, M. E. Cultura Organizacional: identidade, sedução e carisma? 4. ed. Rio de Janeiro: Editora FGV, 2005.

GIL, A. C. Como elaborar projetos de pesquisa. 4. ed. São Paulo: Atlas, 2009.

HAIR JUNIOR, J. F. et al. Análise multivariada de dados. 5. ed. São Paulo. Bookman: 2007.

HOFSTEDE, G. Culture's consequences: international differences in work-related values. Newbury Park: Sage Publications, 1982.

HOFSTEDE, G. et al. Measuring Organizational Cultures: A Qualitative and Quantitative Study Across Twenty Cases. Administrative Science Quarterly, v. 35, n. 2, p. 286-316, 1990. http://dx.doi.org/10.2307/2393392

HOFSTEDE, G. Culturas e organizações: compreender a nossa programação mental. Lisboa: Sílabo, 1997.

JAMES, L. R.; JONES, A. P. Organizational climate: a review of theory and research. Psychological Bulletin, v. 81, n. 12, p. 1096-1112, 1974. http://dx.doi.org/10.1037/ h0037511

JOHNSTON, H. R. A new conceptualization of source of organizational climate. Administrative Science Quarterly, v. 21, p. 95-103, 1976. http://dx.doi. org/10.2307/2391881

KATZ, D.; KAHN, R. Psicologia Social das Organizações. 2. ed. São Paulo: Atlas, 1975.

KOTTER, J. P.; HESKETT, J. L. A cultura corporativa e o desempenho empresarial. São Paulo: Makron Books, 1994.

MAlhotra, N. K. Pesquisa de marketing: uma orientação aplicada. 3. ed. Porto Alegre: Bookman, 2001.

MARTINS, M. C. F. et al. Construção e validação de uma escala de medida de clima organizacional. Revista Psicologia Organizações e Trabalho, v. 4, n. 1, p. 37-60, jan. 2004.

MARTINS, M. C. F. Clima Organizacional. In: SIQUEIRA, M. M. M. (Org.). Medidas do Comportamento Organizacional. Porto Alegre: Bookman, 2008.

MUSIEllO NETO, F. E. A Relação da Cultura Organizacional com a Performance no Setor Comercial do Rio Grande do Norte. In: ENCONTRO DA ASSOCIAÇÃO NACIONAL DE PÓS-GRADUAÇÃO EM ADMINISTRAÇÃO, 26., 2002, Salvador. Anais eletrônicos... Disponível em: <http://www.anpad.org. br>. Acesso em: 10 maio 2009.

OLIVEIRA, M. A. Pesquisas de clima interno nas empresas: o caso dos desconfiômetros avariados. São Paulo: Nobel, 1995.

PETERS, T.; WATERMAN JUNIOR, R. In search of excellence. New York: Harper and How, 1982.

PUENTE-PALACIOS, K. E. Influências das características pessoais e ambientais na avaliação do clima social do trabalho. Revista de Administração, v. 30, n. 3, p. 73-79, jul./set. 1995.

PUENTE-PALACIOS, K. E. Abordagens teóricas e dimensões empíricas do conceito de clima organizacional. Revista de Administração, v. 37, n. 3, p. 96-104, jul./ set. 2002.

QUINN, R. E.; ROHRBAUGH, J. A competing value approach to organizational effectiveness. Public Productivity Review, v. 5, p. 122-140, 1981. http:// dx.doi.org/10.2307/3380029
RICHARDSON, R. J. Pesquisa Social: métodos e técnicas. São Paulo: Atlas, 2008.

RIZZATTI, G. Categorias de análise de clima organizacional em universidades federais brasileiras. 2002. Tese (Doutorado em Engenharia de Produção)-Universidade Federal de Santa Catarina, Florianópolis, 2002. PMid:12109853.

SÁ, M. A. D. et al. O espelho não tem duas faces: um estudo entre a cultura e o clima organizacional na UFPB. In: ENCONTRO DA ASSOCIAÇÃO NACIONAL DE PÓS-GRADUAÇÃO EM ADMINISTRAÇÃO, 30., 2006, Salvador. Anais eletrônicos... Disponível em: <http:// www.anpad.org.br>. Acesso em: 10 maio 2009.

SÁ LEITÃO, J. S.; GUIMARÃES, T. A.; ROSAL, M. A. A. Metodologia de diagnóstico de clima organizacional em ambiente de inovação tecnológica. In: ENCONTRO DA ASSOCIAÇÃO NACIONAL DE PÓS-GRADUAÇÃO EM ADMINISTRAÇÃO, 22., 1998, Foz do Iguaçu. Anais eletrônicos... Disponível em: <http://www.anpad. org.br>. Acesso em: 11 maio 2009.

SANTOS, N. M. B. F. Cultura e desempenho organizacional: um estudo empírico em empresas brasileiras do setor têxtil. Revista de Administração Contemporânea, v. 2, n. 1, p. 47-66, jan./abr. 1998. http://dx.doi.org/10.1590/ S1415-65551998000100004

SANTOS, N. M. B. F. Clima organizacional: pesquisa e diagnóstico. Lorena: Stiliano, 1999.

SANTOS, N. M. B. F. Cultura organizacional e desempenho: pesquisa, teoria e aplicação. Lorena: Stiliano, 2000.

SAVOIE, A.; BRUNET, L. Climat organisationnel et culture organisationnelle: apports distincts ou redondance? Revue québécoise de psychologie, v. 21, n. 3, p. 179-200, 2000.

SCHEIN, E. H. Organizational culture and leadership. 2. ed. San Francisco: Jossey-Bass, 1992.

SCHNEIDER, B. Organizational Behavior. Annual Review of Psychology, v. 36, p. 573-611, jan. 1985. PMid:20809778. http://dx.doi.org/10.1146/annurev. ps.36.020185.003041

SMIRCICH, L. Concepts of culture and organizational analysis. Administrative Science Quaterly, v. 28, p. 339-358, 1983. http://dx.doi.org/10.2307/2392246

SOUZA, E. L. P. Clima e cultura organizacionais: como se manifestam e como se manejam. São Paulo: Edgard Blucher, 1978.

TAMAYO, A. Valores e clima organizacional. In: PAZ, M. G. T.; TAMAYO, A. (Orgs.). Escola, saúde mental e trabalho. Brasília: Editora UnB, 1999.

THÉVENET, M. L'écôt de la mode. Revue Française de Gestion, n. 53-54, p. 19-29, sep./déc. 1985.

VERBEKE, W.; VOLGERING, M.; HESSELS, M. Exploring the conceptual expansions within the field of organizational behaviour: organizational climate and organizational culture. Journal of Management Studies, v. 35, n. 3, p. 303-329, 1998. http://dx.doi. org/10.1111/1467-6486.00095

WALLACE, J.; HUNT, H.; RICHARDS, C. The relationship between organisational culture, organisational climate and managerial values. The International Journal of Public Sector Management, v. 12, n. 7, p. 548-564, 1999. http://dx.doi.org/10.1108/09513559910305339 